\title{
IMPACTO DEL PROYECTO “PODEMOS LEER Y ESCRIBIR” EN EL DESARROLLO DE LA CULTURA ESCRITA EN LAS ESCUELAS LATINOAMERICANAS
}

\author{
Christian Hederich ${ }^{1}$ \\ (con la colaboración de) \\ María Elvira Charria ${ }^{2}$
}

Desde 1995, la secretaría de Educación Pública -SEP- de México, y el Centro Regional para el fomento del Libro en América Latina y el Caribe -CERLALC-, viene adelantado un proyecto educativo de alcance regional, orientado a la formación de lectores y escritores en escuelas latinoamericanas: el Proyecto "Podemos Leer y Escribir"3.

El Proyecto Podemos Leer y Escribir parte del hecho de que todos los países de la región están comprometidos con el mejoramiento de la calidad de la educación haciendo énfasis en la lectura, la escritura y las habilidades matemáticas. Trabajando en ese sentido, los diferentes países dedican enormes esfuerzos a la capacitación de maestros y a la dotación de libros (de texto, de apoyo curricular y recreativos), sin que los resultados de estas acciones sean siempre claros, por la ausencia de seguimiento y evaluación sistemática del proceso.

En este contexto, el Proyecto Leer y Escribir pretende apoyar la tarea de los diferentes gobiernos en el mejoramiento de la calidad de la formación de niños lectores y escritores en las escuelas de educación básica, promover la evaluación de las acciones en el campo y ofrecer herramientas y recomendaciones para la gestión de políticas educativas en es te sentido.

\section{Estrategias y acciones}

Para cumplir con sus objetivos, el proyecto partió de la donación del gobiemo mexicano de un conjunto de acervos bibliográficos. México ha venido adelantando, desde 1986, un programa editorial y de distribución de materiales bibliográficos orientado a favorecer la formación de lectores y escritores en las escuelas públicas, acompañado con algún nivel de capacitación. Este programa, único en la región, se denomina "Rincones de Lectura", y está a cargo de la Unidad de Publicaciones Educativas (UPE) de la Secretaría de Educación Pública.

\footnotetext{
${ }^{1}$ Profesor. Dpto. de Psicopedagogía. Universidad Pedagógica Nacional.

2 Coordinadora del Programa de Lectura. CERLALC.

${ }^{3}$ Se entiende en este contexto la palabra regional refiriéndose a la región de América latina y el Caribe.
} 
A partir de este ofrecimiento, el proyecto seleccionó, dentro de los materiales de la producción de la UPE, un Acervo Bibliográfico Latinoamericano. En la selección de los títulos se buscó un cierto equilibrio fundamentado sobre el logro de la mayor diversidad posible de géneros, estilos y formatos, intentando, eso sí, constituir un conjunto integral y organizado, y no un agrupamiento azaroso de materiales. Para subrayar este último hecho, el proyecto ha buscado promover la noción de "acervo" como unidad que debe usarse, preservarse, y enriquecerse de manera permanente. Cada acervo estuvo compuesto de un total de 93 libros y 5 cintas grabadas, para un total de 98 títulos, de los cuales 47 el programa de Rincones de Lectura tuvo a su disponibilidad inmediata, y 51 títulos que fueron especialmente producidos para el proyecto.

El proceso de entrega de los acervos a cada uno de los países participantes en la experiencia se inició con la identificación de una, o varias, personas u organizaciones, en cada país de la región, que trabajaran en la renovación de las prácticas pedagógicas de la lectura y la escritura. Estas personas o entidades fueron invitadas a participar en el proyecto en calidad de coordinadores nacionales o locales. En cada país los coordinadores decidieron sobre la distribución de los acervos entre las escuelas que se comprometieran, mediante un proyecto pedagógico, con el trabajo en el campo de la lectura y la escritura ${ }^{4}$.

Una vez seleccionados los materiales del acervo, y firmados los convenios internacionales necesarios (SEP México - Autoridades educativas en los países CERLALC), los acervos fueron distribuidos entre los países participantes. Los convenios estipularon el compromiso de las escuelas participantes en la experiencia en la recolección de la información necesaria para la evaluación del impacto del proyecto. Has ta la fecha se han entregado 1035 acervos bibliográficos latinoamericanos en 9 países: Colombia, Costa Rica, Chile, Cuba, El Salvador, Honduras, Nicaragua, Uruguay y Venezuela.

\section{El papel de la evaluación}

Junto con la selección y distribución del acervo bibliográfico latinoamericano, uno de los pilares fundamentales del proyecto reside en la evaluación del proceso y del impacto de los acervos sobre las instituciones participantes en la experiencia.

\footnotetext{
${ }^{4}$ Estos coordinadores no fueron remunerados económicamente por su participación en el proyecto. Sea este el espacio para reconocer y agradecer su importante y desinteresada participación en este esfuerzo. Las coordinaciones estuvieron a cargo de las siguientes personas: Colombia: Martha Cárdenas (Asociación Distrital de Maestros) y Alba Luz Castañeda (Secretaría de Educación de Santafé de Bogotá); Costa Rica: Elizabeth Villalobos (Ministerio de Educación Pública) y Arnobio Maya (SIMED); Chile: Clara Budnick (Ministerio de Educación); Cuba: Georgina Arias (Ministerio de Educación); El Salvador: Martha Gladys de Palacios (Ministerio de Educación); Honduras: Ingrid Cortés (Ministerio de Cultura, Arte y Deporte); Nicaragua: Eduardo Baez (Fundación Libros para Niños) y Yadira Morales (Ministerio de Educación); Uruguay: Rosario Nougues (Federación Uruguaya de Maestros) y Eloisa Salvo ((Ministerio de Educación y Cultura); Venezuela: Wallis de Gómez (Red Lationoamericana de Alfabetización) y Noma Gonzalez (Ministerio de Educación).
} 
Es innecesario justificar a importancia de la evaluación como mecanismo para el mejoramiento de la experiencia. Este era uno de los sentidos que justificaban la importancia de la evaluación, pero no el único. En es te punto hemos creído que la presencia de la evaluación externa del proyecto es fundamental, no solo fuente para la elaboración de recomendaciones de política, ni para la construcción de conocimiento sobre el tema, sino también como vitalizador de la experiencia misma. En es te último sentido, la evaluación se ha considerado tam bién como una forma para el acompañamiento de las escuelas participantes, y como mecanismo de formación y capacitación de maestros y autoridades educativas.

Conceptuar el proceso evaluativo como forma de acompañamiento puede parecer extraño, o al menos inusual, por lo que me permitiré explicarlo brevemente. Dentro de una tradición mecanicista, el proceso evaluativo ha sido considerado como retroalimentación de la experiencia para la toma de decisiones políticas que conducirían a la cualificación y optimización de su impacto. Este sentido de la evaluación esta presente en el proyecto, pero no es el único sentido. Dentro de otra óptica, la evaluación puede representar un punto de contacto entre escuelas en torno a un proyecto común, orientado al mejoramiento de la calidad de su labor. En este sentido, la evaluación pemite a la institución hacerse visible a los ojos de las otras instituciones, cercanas y lejanas, participantes también en la experiencia. Así, la evaluación es parte del diálogo necesario entre las instituciones, y cons tituye un espejo que permite, a cada cual, conceptualizarse en su desarrollo frente a un proyecto común.

Ahora, la evaluación también ha tenido su parte en la formación y capacitación de las comunidades educativas. Para ello, dirigimos una serie de encuestas que debían ser respondidas por diferentes participantes de la comunidad educativa: director escolar, coordinador del proyecto en la escuela, maestros, alumnos y padres. En cada una de estas encuestas, además de que se cumplía con el objetivo de infomar a cada cual acerca de la experiencia misma, las mismas preguntas sugerían actividades y posibilidades para el mejor aprovechamiento de los materiales. Así, al tiempo que el maestro informaba sobre su aproximación a los materiales del acervo, encontraba sugerencias hacia la promoción de su uso, las actividades a realizar con los materiales, etc. Esto ocurría de forma similar en todas las encuestas.

La experiencia de los diferentes países fue recogida en reuniones internacionales celebradas en México. Durante estas reuniones, se expusieron los resultados del análisis regional así como las experiencias particulares de los diferentes países, e incluso de algunas escuelas específicas, durante el desarrollo del proyecto. Hasta el momento dos reuniones se han celebrado que coinciden con dos momentos de análisis de la experiencia.

Concluyendo, desde una perspectiva espistemológica consideramos que la evaluación no ha sido un componente extraño al éxito del proyecto, sino que, por el contrario, explica en buena medida los logros. El "observador", ha tenido su parte en el logro del "observado". El resultado de un compromiso cada vez mayor 
con esta forma de conceptualizar el proceso evaluativo ha sido un viraje progresivo hacia formas cada vez más participativas de la evaluación. Expondremos estos avances más adelante, cundo tratemos la prospectiva del proyecto. Antes, deberemos explicar la forma en que se ha elaborado el análisis de la experiencia, y algunos de los principales resultados.

\section{La naturaleza del análisis}

El objetivo último de la entrega de los acervos, y de la experiencia misma es un objetivo de naturaleza cultural: la apropiación de la cultura escrita por parte de cada uno de los participantes en la experiencia.

Para dimensionar las dificultades de esta empresa, debemos considerar que la población atendida en las escuelas públicas, en la mayoría de nuestros países, está en su inmensa mayoría culturalmente alejada de la escritura en su cotidianidad familiar. En esta situación, para muchos niños los modelos lectores y escritores más determinantes sólo se están viviendo en la escuela.

Ahora bien, si es la escuela la que representa la cultura escrita en la cotidianidad de los estudiantes, debe por otro lado reconocerse que, de manera general, el modelo de escuela que ha prevalecido en la región está lejos de la cultura escrita, tanto des de el punto de vista administrativo, como del punto de vista pedagógico. Así, una buena parte de las escuelas latinoamericanas permanecen de por sí alejadas de la cultura escrita, y una buena parte de los maes tros, incluso aquellos dedicados a la ens eñanza de la lectura y la escritura no son, en sí, lectores y escritores.

De esta manera, al acercarnos a analizar los efectos de la llegada de los libros, debemos considerar, no sólo los indicadores del comportamiento lector de los alumnos, sino también los indicadores de avance en la familia, además de examinar los logros del programa en la construcción de cultura escrita en la escuela, desde la escuela, y como escuela. Esto es, los avances del maestro como lector, los avances en la confomación del aula como espacio de lectura (avances pedagógicos en la lectura), y los avances de la escuela en relación con la capacidad de colocar el acervo a disponibilidad de los participantes y mantenerlo.

Así, nos encontramos con un análisis, que por su naturaleza, concibe como logros las aproximaciones de alumnos, familias, maestros e instituciones a la cultura de lo escrito. Algunos de estos logros, a su vez, pueden ser considerados como factores potencialmente asociados con otros niveles de logro. Por ello, nuestra concepción del logro es más bien holística: los diferentes entornos se aproximan a la cultura de lo escrito, y los niveles de proximidad alcanzados en unos niveles son, al tiempo, factores que en otros niveles resultan condiciones necesarias.

La presentación general de los resultados del proyecto en los diferentes niveles de anális is para los diferentes actores participantes en la experiencia escapa a los 
alcances del presente artículo. En lo que sigue, presentaremos algunos de los principales resultados obtenidos durante la segunda etapa del análisis.

\section{Método}

\section{Población y muestra}

En términos de las escuelas participantes, se buscó recoger información sobre la totalidad de las instituciones participantes en el proyecto, aunque esté logro no fue posible por las dificultades inherentes a la coordinación de un conjunto tan diverso de tiempos escolares.

La información disponible, para la segunda etapa, fue recogida de un total de 483 instituciones en 6 (seis) países: Colombia, Costa Rica, Cuba, Honduras, Nicaragua y Venezuela.

\section{Muestra efectiva (segundo momento del análisis)}

\begin{tabular}{|c|c|c|c|c|c|c|c|c|c|}
\hline \multirow[b]{2}{*}{ PAÍS } & \multicolumn{2}{|c|}{$\begin{array}{l}\text { Institucion } \\
\text { es }\end{array}$} & \multicolumn{2}{|c|}{ Maes tros } & \multicolumn{2}{|c|}{ Niños } & \multicolumn{2}{|c|}{ Padres } & \multirow{2}{*}{$\begin{array}{l}\text { Número } \\
\text { de } \\
\text { acervos }\end{array}$} \\
\hline & $\bar{N}$ & $\%$ & $\mathrm{~N}$ & $\%$ & $\mathrm{~N}$ & $\%$ & $\mathrm{~N}$ & $\%$ & \\
\hline Colombia & 135 & 28.0 & 429 & 26.8 & 751 & 32.1 & 418 & 23.3 & 200 \\
\hline Costa Rica & 34 & 7.0 & 0 & 0.0 & 0 & 0.0 & 0 & 0.0 & 50 \\
\hline Cuba & 144 & 29.8 & 742 & 49.4 & 631 & 27.0 & 598 & 33.3 & 150 \\
\hline Honduras & 37 & 7.7 & 29 & 1.8 & 43 & 1.8 & 38 & 2.1 & 50 \\
\hline Nicaragua & 81 & 16.8 & 169 & 10.6 & 192 & 8.2 & 156 & 8.7 & 135 \\
\hline Venezuela & 52 & 10.8 & 230 & 14.4 & 720 & 30.8 & 587 & 32.7 & 200 \\
\hline Total & 483 & $\begin{array}{l}100 . \\
0\end{array}$ & $\begin{array}{l}159 \\
9\end{array}$ & $\begin{array}{l}100 . \\
0\end{array}$ & 2337 & $\begin{array}{l}100 . \\
0\end{array}$ & $\begin{array}{l}179 \\
7\end{array}$ & $\begin{array}{l}100 . \\
0\end{array}$ & 785 \\
\hline
\end{tabular}

En el caso de Costa Rica, contamos con información de las instituciones, pero no tenemos información de maestros, padres y alumnos, en tanto que los tiempos para adelantar la tarea en el país no lograron coincidir con los del presente anális is 5 .

La ausencia en la muestra de algunos países participantes del proyecto obedece a dos razones: por un lado, razones de tipo técnico que no logramos resolver hasta el momento del análisis, nos han impedido que la información de Uruguay, que corresponde a 73 escuelas, 226 maestros, 178 niños, 166 padres,

\footnotetext{
${ }^{5}$ En un tipo de trabajo regional como el presente, en el que nos estamos insertando en los desarrollos educativos de los países, y no construyendo un propio equipo del proyecto, es fundamental comprender la dificultad para obtener la misma información, en el mismo tiempo, y con las mismas condiciones en todos los países. Debe considerarse un logro grande en la ejecución del proyecto, el propio hecho de haber logrado obtener la información con la que hoy se cuenta para este estudio.
} 
sea incorporada; de otro lado, nos encontramos con la ausencia de El Salvador y Chile, países en donde, en el momento de elaborar el análisis, el acervo llevaba menos de un mes de estar en las escuelas.

Variables

\begin{tabular}{|c|c|}
\hline $\begin{array}{ll}\text { Nivel de } \\
\text { análisis }\end{array}$ & Variables \\
\hline Escuela & $\begin{array}{l}\text { - Cubrimiento del trabajo en la escuela; cuantificación de la } \\
\text { población participante. } \\
\text { - Localización, clasificación y control de los acervos, como un } \\
\text { paquete de tres variables que hablan del acceso real a los libros } \\
\text { y el cuidado y conservación de los mismos, y que hemos } \\
\text { englobado en el concepto general de disponibilidad. } \\
\text { - Participación de diversos miembros de la comunidad escolar en } \\
\text { tareas para facilitar el acceso y cuidado de los libros. } \\
\text { - El aumento de las colecciones y las formas de lograrlo. }\end{array}$ \\
\hline Maestros & $\begin{array}{l}\text { - El número y tipo de libros que señala haberle interesado } \\
\text { - La diversidad de estrategias que usa en el trabajo con los libros } \\
\text { - El tipo de capacitación que ha recibido }\end{array}$ \\
\hline Alumnos & $\begin{array}{l}\text { - El número de libros que ha leído, indicado por el número de } \\
\text { títulos que es capaz de recordar como de su gus to o disgusto } \\
\text { - Los lugares donde los lee } \\
\text { - El tipo de libros que le gustan y le disgustan y las razones que } \\
\text { explica para ello } \\
\text { - La lectura de algunos de estos libros por parte de su familia } \\
\text { Las recomendaciones que da para mejorar el préstamo en la } \\
\text { escuela. }\end{array}$ \\
\hline Padres & $\begin{array}{l}\text { - El número de libros que ha leído -retomado por el número de } \\
\text { libros que es capaz de recordar como de su gusto o disgusto } \\
\text { - Los lugares donde los lee }\end{array}$ \\
\hline
\end{tabular}

La naturaleza diversa de las variables ha invitado a un trabajo que ha implicado tanto un análisis cuantitativo, como una mirada cualitativa de otro tipo de información para una descripción y comprensión de la situación. Hemos trabajado su descripción, y comprensión, pero a la vez hemos buscado relaciones cuantitativas entre algunas de estas variables.

\section{Resultados}

\section{Cobertura del programa}

De acuerdo con los datos disponibles, la cobertura actual del proyecto es verdaderamente significativa. En total, en las 441 instituciones sobre las cuales tenemos información, pueden distinguirse 159.796 alumnos, 6.184 maestros, 
1.108 directivos docentes, y 11.641 adultos no maestros, directamente participantes en la experiencia. En promedio, cada institución vincula con el proyecto a poco más de 341 alumnos, 14 maestros, y más de 2 directivos (entre los que se cuentan rectores y coordinadores académicos).

Calculada la proporción de la institución que directamente participa en esta experiencia, los resultados indican que casi el $80 \%$ de los maestros y el $86.6 \%$ de los alumnos están participando activamente en el programa. Estas cifras muestran una proporción muy alta de la población escolar que se encuentra vinculada con la experiencia El valor del $100 \%$ de esta relación, corresponde a un proyecto generalizado que se hace institucional, con mayores probabilidades de que pueda pervivir e irse incorporando en el proyecto pedagógico fundamental de la institución.

\section{La disponibilidad: un logro institucional}

Con variaciones y particularidades, cuando una colección de libros llega a la escuela latinoamericana ésta es vista como la llegada a casa de un regalo muy deseado, que percibimos como muy hermoso e importante, pero con el cual no tenemos un contacto cercano en nuestro contexto cultural. Puede ocurrir que, aunque le admiremos, empecemos por ponerlo en lugares en los que nos estorba, o en sitios poco prácticos para encontrarlo. Finalmente, a medida que aumente nuestro contacto con los libros ocurrirá que le iremos encontrando el lecho perfecto que lo incluya en nues tro hogar.

Buena parte de las dificultades iniciales con los libros se explican por una tensión fundamental, siempre presente en la escuela, que hemos denominado la tensión uso-permanencia. Por un lado, si los libros se usan, se leen y se prestan, ocurre que en mayor o menor medida es tos se dañan y se pierden. Al ser parte del "inventario escolar", esto le implica al responsable del mismo su reposición. Para evitar estas pérdidas, los libros con frecuencia se mantienen encerrados, y se restringe su acceso y su uso. En la medida en que no hay préstamo, los libros no se despastan y menos se pierden pero, claro, bajo la condición de que no se usen. En cualquiera de estos dos casos los libros dejan de estar disponibles a la comunidad: bien por que se han perdido, o bien porque no hay acceso a los mismos, lo cual al final, como dice la canción, "...no es lo mismo, pero es igual”.

Así hemos concebido la disponibilidad, como un equilibrio entre los dos polos de la tensión entre el uso y la permanencia, según el cual los materiales pemanecen completamente accesibles a todos los participantes, intentando prolongar su vida útil hasta el máximo de las posibilidades. El logro de la disponibilidad es un reto institucional que con frecuencia es difícil de conseguir en las escuelas a las que han llegado libros, y lo hemos considerado como la medida institucional del logro más importante. A fin de hacer operacional el concepto de disponibilidad, dentro del análisis se examinaron en detalle los indicadores de 1] ubicación de los 
materiales en la escuela; 2] la clasificación de los mismos; y 3] el control del préstamo.

Presentar una análisis detallado de la disponibilidad lograda por las escuelas consideradas en la muestra en cada uno de estos indicadores queda por fuera de las posibilidades de espacio en el presente artículo. Baste mencionar que los resultados indican una alta disponibilidad de los materiales en las escuelas, directamente relacionada con el tiempo que lleva la escuela de trabajo con el proyecto.

En el momento del segundo análisis, las instituciones consideradas para el mismo habían mantenido el acervo entre tres meses, para el caso de las instituciones de Honduras y Venezuela, y 15 meses para el caso de las instituciones de Colombia, Cuba y Nicaragua. En estos términos contamos con información de 123 escuelas (25\% del total) que llevaban en ese momento tres meses con sus acervos, frente a 360 escuelas (75\%) que llevaban 15 meses, un año más, desde que iniciaron su proceso.

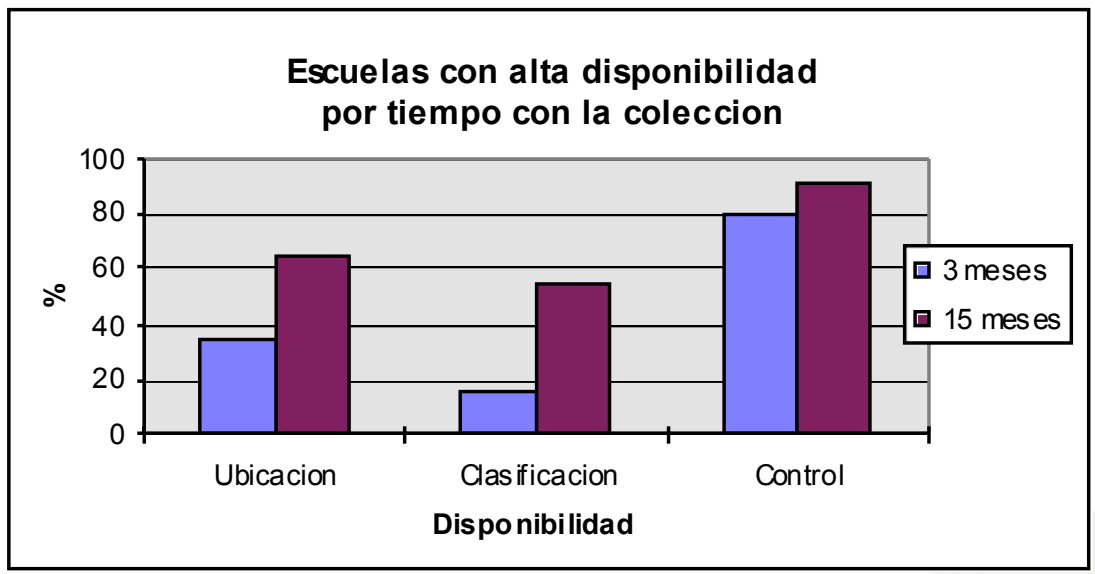

Los resultados muestran una relación directa y altamente significativa, en las tres dimensiones examinadas, entre los niveles de disponibilidad del acervo y la trayectoria de la institución en el proyecto. Cuando el acervo llega a la escuela, llega posiblemente como un elemento extraño, para el cual no hay un lugar definido, y con el cual no se sabe con claridad qué puede o debe hacerse. En muchos casos, por la naturaleza de la tradición de la administración educativa en la región, la colección es considerada como algo valioso y costoso que debe ser preservado a toda costa, aunque ello implique no utilizarlo. Por esta razón, en ausencia de un espacio previamente adecuado para cumplir con las funciones de biblioteca, los libros son guardados en el lugar que se considera más seguro: la dirección escolar. En este contexto, la organización de los libros puede no ser considerada necesaria, o bien ser hecha con criterios apropiados para el adulto. No es frecuente en esta situación que los materiales se presten a los maestros, alumnos o padres. Cuando existe préstamo, el control de él puede tomar dos formas: la primera, establecer un rígido control, con múltiples requisitos que aseguren la devolución del libro; o la segunda, no establecer control, en la medida 
en que la relación directa de autoridad garantizaría graves repercusiones a la falta. Este un manejo altamente centralizado y jerarquizado del recurso, caracterizado por muy bajos niveles de disponibilidad de los materiales, tanto en lo relacionado con su ubicación, como con su clasificación y control.

En la medida en que el acervo permanece dentro de la institución y los maestros lo trabajan, los libros empiezan a ser solicitados por niños y padres, lo cual seguramente presionará por una mayor dis ponibilidad inmediata de los libros, lo que a su vez hará patente la necesidad de su mantenimiento, así como de un control más eficiente y sistemático del préstamo. El resultado: progresivamente las instituciones incrementan la disponibilidad, inmediata y a futuro, de la colección. Se esta, acá, incorporando la escuela en la cultura de lo es crito.

\section{Alumnos}

\section{Libros leídos}

Aunque el número de libros leídos no determina de ninguna manera definitiva al lector, sí sabemos que sólo en la medida en que el lector se encuentre con más y más libros tendrá mejores posibilidades de encontrar aquel o aquellos libros que verdaderamente podrán conmoverlo internamente y a partir de esta experiencia, consolidar una relación con la palabra impresa. Por ello, el número de libros leídos es el mejor descriptor de un lector en proceso de avance dentro de la cultura de lo escrito.

El número de libros leídos por los niños dentro de la colección del acervo fue obtenido por la cantidad de títulos específicam ente referenciados por el niño como de su gusto o disgusto dentro de la colección. Conocemos que ello está limitando el número de libros que consideramos leídos a aquellos que el sujeto recuerda y que, en muchos casos, entendiendo el efecto del tiempo sobre el recuerdo, pueda ser que no se recuerde todo lo leído. Sin embargo, nos ha parecido que, tomado así, el dato es la manera más fiable de aproximarnos a conocer el número de libros leídos.

Los resultados indican un promedio de poco más de 4.71 libros leídos por niño. Sólo 89 de los niños escuestados (3.8\%) no reportaron ningún título, y 81 (3.5\%) referenciaron más de 10 títulos. 


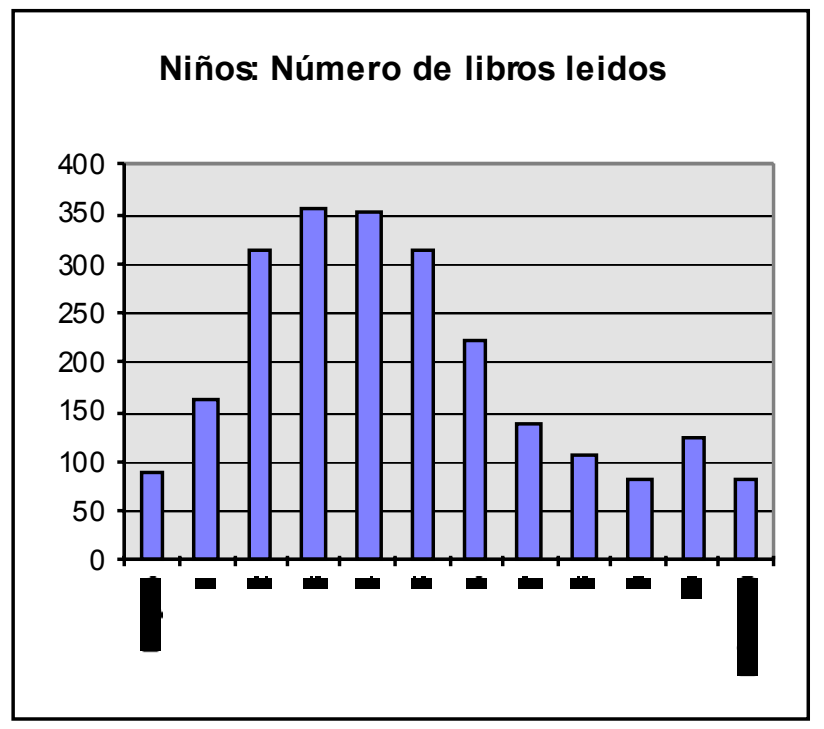

\begin{tabular}{|c|rc|}
\hline $\begin{array}{c}\text { Número de títulos } \\
\text { referenciados por } \\
\text { los niños }\end{array}$ & $\begin{array}{r}\text { Reportes } \\
\text { de niños }\end{array}$ & \\
\hline Ninguno & 89 & 3.8 \\
1 & 164 & 7.0 \\
2 & 313 & 13.4 \\
3 & 355 & 15.2 \\
4 & 352 & 15.1 \\
5 & 312 & 13.4 \\
6 & 222 & 9.5 \\
7 & 140 & 6.0 \\
8 & 105 & 4.5 \\
9 & 81 & 3.5 \\
10 & 123 & 5.3 \\
Más de 10 & 81 & 3.5 \\
\hline Total & 2337 & 100.0 \\
\hline
\end{tabular}

El bajo porcentaje de niños que reporta no haber leído libros del acervo es notorio, si tenemos en cuenta que la muestra ha tenido una alta participación de alumnos de primer grado. Sabemos que aunque hoy en día comprendamos que se espera que la totalidad de los niños tengan alguna experiencia lectora desde tem pranas edades, la escuela tradicionalmente no ha facilitado el encuentro de los alumnos con los libros sino hasta que "saben leer" y ello corresponde, en la jerga de la escuela, básicamente a partir del segundo grado. Este dato nos permite aventurar que, en las escuelas del estudio, el libro ha encontrado lectores aún entre los más pequeños y que son pocos, aunque no despreciable en número, los niños que en el plazo de tiempo que el acervo lleva en sus escuelas no han leído.

La posibilidad de reconocer relaciones placenteras o displacenteras frente al encuentro con los diversos textos es una de las características de los lectores. Así, el hecho de que un porcentaje de los alumnos que registraron información estén en posibilidad de hacerlas explícitas nos está indicando un avance en su comportamiento lector, pocas veces atendido en la tarea escolar en la que casi siempre se trabaja con un libro y todos los alumnos deben interesarse por él, cerrando de alguna manera la expresión de los sentires de los alumnos frente al material ${ }^{6}$.

\footnotetext{
${ }^{6}$ Las razones aducidas por los alumnos a su gusto o disgusto frente a al gunos libros fueron examinadas en detalle, $\infty$ n resultados muy interesantes. Para mayores detalles, consúltese el informe técnico.
} 


\section{Recuadro}

\begin{tabular}{|l|ll|}
\hline $\begin{array}{l}\text { No. de libros que } \\
\text { disgustan }\end{array}$ & $\mathrm{N}$ & $\%$ \\
\hline 0 & 2031 & 86.9 \\
1 & 190 & 8.1 \\
2 & 63 & 2.7 \\
3 & 53 & 2.3 \\
\hline Total & 2337 & 100 \\
\hline
\end{tabular}

Entre los títulos referenciados por los muchachos, la enorme mayoría eran mencionados como libros que les habían gustado. Existen 306 niños (13.1\%) que lograron manifestar su disgus to por algún o algunos de los libros que habían leído: 190 lo manifiestan frente a un libro, y 116 por dos o tres, y exponen razones para ello. Este es un indicador de desarrollo del comportamiento lector nada despreciable.

\section{Lugares de lectura}

A medida en que se avanza en el proceso lector, la actividad lectora es cada vez más un acto personal-individual y en menor grado, un acto compartido-social. La lectura en el aula es una actividad compartida y social que tiene connotaciones específicas. En general, es una lectura dirigida y controlada por el maestro, con claros objetivos que definidos por él en función de sus objetivos de enseñanza, sin que necesariamente estos coincidan con los del alumno; allí la lectura tiene fundamentalmente connotaciones de actividad social. Fuera del aula, el alumno puede ejercer la lectura como acto individual y personal, de acuerdo a sus objetivos aunque también encontrará algunas ocasiones en las que tenga y/o quiera ejercer la lectura de los libros del acervo como actividad grupal. Las posibilidades de ejercer la lectura en el aula y fuera de ella, permiten considerar que, al menos potencialmente, este lector puede acercarse a ellos de manera individual y social. Aunque los datos registrados no permiten explorar el tipo de relaciones lectoras -sociales o individuales- establecidas por los alumnos, vale la pena examinarlo y explorar sus relaciones con el número de libros leídos.

De los 2.248 niños sobre los que obtuvimos información, $654(29 \%)$ no reportaron específicamente ningún lugar de lectura. Descontando éstos, la gran mayoría de los niños dijeron haber leído sólo en el aula (37.2\%), el 18.7\% haberlo hecho sólo en la biblioteca, y el $14.1 \%$ haberlo combinado entre el aula y la casa. Sólo 60 niños, que representan el 3.8\% dicen haber leído en los tres lugares.

Las relaciones entre los lugares de lectura del niño y el promedio de los libros que referencia son bas tante interesantes.

Primero, examinado cada lugar por separado, se observa que los niños que manifiestan haber leído en la biblioteca o en la casa muestran un promedio 
significativamente mayor de títulos leídos que aquellos que no leyeron en estos lugares. Esta relación no se observa respecto de la lectura en el aula.

Segundo, y de forma consistente con lo anterior, se observa que los niños que manifiestan haber leído en dos o tres de los lugares propuestos muestran un número significativamente mayor de títulos leídos que aquellos que sólo mencionan un lugar.

Los resultados son claros en indicar que, si bien la lectura en el aula puede ser una buena introducción al mundo del libro, en la medida en que los niños independizan su lectura de la actividad del aula y la ejercen en la biblioteca o en la casa, además de lo que esto significa en términos de su desarrollo como lectores, muestran también un número mayor de títulos leídos.

\section{Recuadro}

\begin{tabular}{|c|c|c|c|c|c|c|c|c|c|}
\hline & \multicolumn{3}{|c|}{ Lectura en el aula } & \multicolumn{3}{|c|}{ Lectura en biblioteca } & \multicolumn{3}{|c|}{ Lectura en casa } \\
\hline & $\begin{array}{l}\text { Promedi } \\
\text { de libr } \\
\text { leídos }\end{array}$ & $\begin{array}{l}\text { Desv. } \\
\text { St. }\end{array}$ & Casos & $\begin{array}{l}\text { Promec } \\
\text { de li } \\
\text { leídos }\end{array}$ & $\begin{array}{l}\text { Desv. } \\
\text { St. }\end{array}$ & asos & $\begin{array}{l}\text { Promedi } \\
\text { de libr } \\
\text { leídos }\end{array}$ & Desv. & \\
\hline$\overline{N O}$ & 4.6669 & 3.1604 & 1273 & 4.2543 & 2.8700 & 1691 & 4.5062 & 3.0 & 1780 \\
\hline SI & 4.7585 & 2.9118 & 1064 & 5.8978 & 3.1841 & 646 & 5.3555 & 2.9987 & 557 \\
\hline Total & 4.7086 & 3.0494 & 2337 & 4.7086 & 3.0494 & 2337 & 4.7086 & 3.0494 & 2337 \\
\hline
\end{tabular}

Examinado cada lugar por separado, se observa que los niños que manifiestan haber leído en la biblioteca o en la casa muestran un promedio significativamente mayor de títulos leídos que aquellos que no leyeron en estos lugares. Esta relación no se observa respecto de la lectura en el aula.

\begin{tabular}{|llll|}
\hline $\begin{array}{l}\text { No. } \\
\text { lugares } \\
\text { lectura }\end{array}$ & $\begin{array}{l}\text { de } \\
\text { deromedio Desv. }\end{array}$ & Casos \\
de libros St. & \\
\hline 1 & $\begin{array}{llll}\text { leídos } \\
2.9202\end{array}$ & 2.9964 & 1040 \\
2 & 5.6822 & 2.8087 & 494 \\
3 & 6.2500 & 3.2554 & 60 \\
\hline Total & 5.2064 & 2.9756 & 1594 \\
\hline
\end{tabular}

Los datos sobre la diversidad de los lugares en que los niños leen los libros mues tran que cerca del $65 \%$ manifiestan haber leído sólo en un lugar, $31 \%$ en dos lugares, y sólo cerca del $4 \%$ muestran haber leído en los tres lugares propuestos. Los resultados indican que, a mayores lugares de lectura, mayores promedios de lectura.

\section{Maestros}

En general, la actividad que con mayor frecuencia proponen los maestros a sus alumnos es la de lectura en voz alta (82\%). Las actividades de escritura son propuestas por el $65 \%$ de los maestros, le siguen en orden descendente los 
trabajos de exploración del material $(50 \%)$, la creación de cuentos colectivos $(47 \%)$, y otras ${ }^{7}(27 \%)$.

No es posible señalar una sola actividad pedagógica como la que verdaderamente promueve el uso de los libros por encima de otras. Así como los libros son diversos, los momentos, los espacios, y los propósitos para leer, también lo son. Es por ello, que hemos de considerar que la variedad de estrategias usadas por el maestro puede ser un indicador de logro, en tanto, estará con ello posibilitando a los alumnos diversos tipos de encuentros con los libros, y a la vez desarrollando en ellos variedad de aspectos de las habilidades que involucran los actos lectores.

En relación con la diversidad de actividades, sólo 34 maestros $(2.1 \%)$ dicen no haber ejecutado ninguna actividad, y una minoría (13.7\%) mencionan sólo una actividad, con frecuencia, la lectura en voz alta. En contraste, más del $55 \%$ de los maestros reportan realizar al menos tres tipos diferentes de actividad con los libros. Los resultados indicaron que, a mayor diversidad de actividades de los maes tros, mayores promedios de lectura por parte de sus alumnos.

\section{Recuadro}

\begin{tabular}{|l|ll|}
\hline Actividad & $\begin{array}{l}\text { Número } \\
\text { de } \\
\text { reportes }\end{array}$ \\
\hline Lectura en voz alta & 1310 & $82 \%$ \\
Actividades de escritura & 1033 & $65 \%$ \\
Exploración libre de los libros & 806 & $50 \%$ \\
Creación de cuentos & 759 & $47 \%$ \\
Otras actividades & 425 & $27 \%$ \\
\hline Total & 1599 & 100 \\
\hline
\end{tabular}

\section{Gráfica}

\begin{tabular}{llll}
\hline $\begin{array}{l}\text { Numero de } \\
\text { actividades } \\
\text { diferentes }\end{array}$ & $\begin{array}{l}\text { Promedios } \\
\text { de lectura }\end{array}$ & D.S & Casos \\
\hline 1 & 4.40 & 2.37 & 61 \\
2 & 4.91 & 2.66 & 192 \\
3 & 5.11 & 2.67 & 190 \\
4 & 5.10 & 2.45 & 168 \\
5 o más & 5.53 & 2.55 & 55 \\
\hline
\end{tabular}

\footnotetext{
${ }^{7}$ Estas últimas respuestas fueron rastreadas, una por una, y posteriormente categorizadas. Al respecto, veáse el informe técnico del segundo momento de análisis, publicado en la hoja web del Cerlalc: www.cenlalc.com.
} 


\section{Padres}

De acuerdo con los datos, un elevado porcentaje de padres (94\%), relacionan al menos un título del acervo, obteniéndose un promedio de lectura del acervo sorprendentemente alto (3.58). Sólo 107 padres (6\%) reportan no haber leído ninguno de los títulos de la colección, lo que resulta ser significativamente bajo.

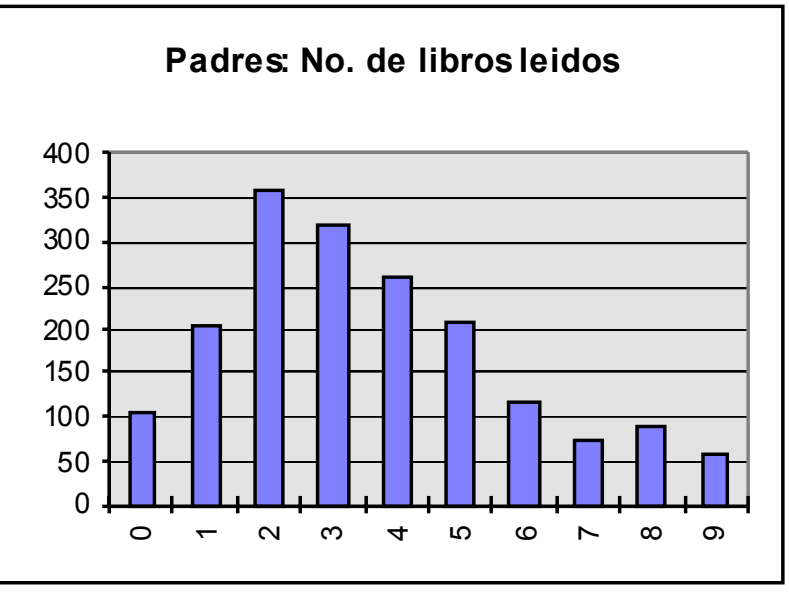

\begin{tabular}{|c|rr|}
\hline $\begin{array}{c}\text { Número de } \\
\text { libros leídos }\end{array}$ & N & \multicolumn{2}{|c|}{$\%$} \\
\hline 0 & 107 & 6.0 \\
1 & 205 & 11.4 \\
2 & 358 & 19.9 \\
3 & 318 & 17.7 \\
4 & 262 & 14.6 \\
5 & 206 & 11.5 \\
6 & 117 & 6.5 \\
7 & 74 & 4.1 \\
8 & 90 & 5.0 \\
9 o más & 60 & 3.3 \\
\hline Total & 1797 & 100 \\
\hline
\end{tabular}

Sólo cerca del $20 \%$ manifiesta haber leído en la escuela. Los padres que leyeron en casa muestran un mayor promedio de libros leídos (3.82) que aquellos que no lo hicieron (2.85).

\begin{tabular}{|l|ll|}
\hline Lugares de lectura & \multicolumn{2}{|l|}{$\begin{array}{l}\text { No. de \% } \\
\text { padres }\end{array}$} \\
\hline Lectura en casa & 1320 & 88.9 \\
Lectura en escuela & 282 & 19.0 \\
Lectura en otro lugar & 32 & 2.2 \\
\hline
\end{tabular}

Tal y como se espera, la gran mayoría de los padres $(90 \%)$ dicen haber leído los títulos del acervo en la casa. La lectura de los padres en su casa, vista como posibilidad de llegada del libro al hogar, con todo lo que ello significa para el niño en relación con su posibilidad de observar y/o compartir momentos lectores en familia, es importante para favorecer sus desarrollos lectores, y constituye un logro importante del proyecto.

Por otro lado, vale mostrarse sorprendido ante el dato que el $19 \%$ de los padres indican haber leído en la escuela. Aunque sea una minoría todavía la que lee en la escuela, se trata no ya de lecturas que los padres han adelantado en la mayor parte de las veces promovidas por los hijos que posibilitan el encuentro con el libro al llevarlo a la casa, sino de lecturas que posiblemente se han dado en el marco de su acercamiento al local escolar para reuniones, y que le dan la posibilidad al padre de una elección personal del libro. Es un logro escolar de gran importancia para las escuelas de nuestra región abrir las puertas de la escuela a los padres para invitarlos a encontrarse con los libros 


\section{Conclusiones y prospectiva}

En síntesis, el proyecto "Podemos leer y escribir" se perfila como un esfuerzo con altos niveles de impacto en la población atendida. Los alumnos leen los títulos del acervo y construyen sus propias posiciones sobre los materiales leídos. En buena parte esto se ve favorecido por maestros que implementan diferentes actividades con esos libros. Los alumnos, por su parte llevan los títulos a sus casas y estos títulos son leídos por sus padres. Asistimos en estas escuelas a un proceso de progresiva aproximación cultural a la lengua escrita.

Esta dinámica cultural, de aproximación a los escrito se presenta, con particular intensidad, en escuelas que dan alta disponibilidad a los materiales: que los ubican en sitios accesibles para todos, que los clasifican, los prestan, los reparan, y mantienen un control claro y sencillo del préstamo. Esta ganancia de disponibilidad es, insistimos, progresiva: entre más tiempo lleva la escuela participando en el proyecto, mayor es la disponibilidad de sus materiales.

Cabe preguntarse por las razones que explican estos altos niveles de impacto. Varias razones ya han sido expuestas: 1] la calidad, diversidad y pertinencia de los materiales, 2] la estrategia de distribución de los mismos, que se iniciaba en la elección de coordinadores nacionales y locales y que estimulaba la formulación de proyectos pedagógicos institucionales orientados hacia la lectura y la escritura-, y 3] el acompañamiento derivado de la evaluación y el seguimiento de la experiencia.

Todas estas razones podrían apuntar en la misma dirección: a pesar de las limitaciones de recursos, el proyecto "Podemos Leer y Escribir" ha mantenido una motivación básica en sus participantes a través de la conformación de una red de trabajo pedagógico que, de muchas maneras, se mantiene, se retroalimenta y se perfecciona en el logro de sus objetivos. Sin esa motivación básica, el proyecto carecería de la base que le da unidad y sentido.

Para intentar fortalecer esta dinámica, se ha propuesto un perfeccionamiento de la evaluación y seguimiento de forma que, a través de un proceso de autoevaluación, las comunidades escolares identifiquen con mayor claridad sus logros y carencias frente a su trabajo en la lectura y la escritura.

Esta propuesta está siendo muy bien acogida en todos los países ${ }^{8}$ que empiezan a trabajarla con los directivos, supervisores y maestros Podemos suponer que, de lograrse este tipo de proceso en las escuelas participantes, los objetivos del proyecto se habrán cumplido en su totalidad. Las escuelas habrán iniciado un proceso de aproximación a la cultura escrita ya prácticamente irreversible. en el que toda la comunidad estará íntimamente comprometida.

\footnotetext{
${ }^{8}$ Los proceso de autoevaluación se adelantarán $\infty n$ la participación en proyecto de nuevos países en los que además de las escuelas incorporaremos a Escuelas Normales e Institutos de Formación Docente. Los países que trabajan ya en el proyecto son: México, Honduras, Guatemala, El Salvador, Nicaragua, Costa rica, Panamá Colombia, Venezuela, Cuba, Chile, Uruguay 